\title{
RESEARCH
}

Open Access

\section{Obstructive sleep apnoea is associated with dynamic intra-thoracic central airway collapse: results of a 10-year multi-centre retrospective analysis}

Thomas David Crowhurst ${ }^{1,2,3^{*}}$ (D) Petar Tcherveniakov², Brett Lorraine ${ }^{2}$, Jonathan Frederick Polasek ${ }^{1,2}$, Phan Tien Nguyen ${ }^{1,2}$ and Aeneas Yeo ${ }^{1,2}$

\begin{abstract}
Background: Disproportionate bulging of the pars membranosa into the central airway on expiration, now termed 'excessive dynamic airway collapse' (EDAC), has poorly characterised significance. Despite physiological arguments supporting an association between obstructive sleep apnoea (OSA) and dynamic intra-thoracic central airway collapse, this is unproven.

Methods: An audit was conducted of patients having had both a dynamic $\mathrm{CT}$ chest and a diagnostic polysomnogram between 2009 and 2018 and within 2 years of one another. Dynamic airway collapse was quantified by the presence of EDAC ( $\geq 75 \%$ collapse) at any of five sites plus a composite index of mean crosssectional area (CSA) change. Linear regression models were used to assess associations between pre-defined variables.
\end{abstract}

Results: A total of 59 patients was included. Median apnoea-hypopnoea index (AHI) was 13 events/hour (IQR: 5-31 events/hour | range 0-153 events/hour). EDAC was not identified. Median composite dynamic airway collapse index was 18\% (IQR: 9-30\% | range: 0-63\%). Unadjusted and adjusted linear regression analyses demonstrated statistically significant associations between degree of dynamic intra-thoracic central airway collapse and multiple OSA indices. In the adjusted model, every $10 \%$ worsening in dynamic airway collapse was associated with an increase in apnoea-hypopnoea index (AHI) of 5 events/hour (95\% confidence interval: 0.9-10 events/hour $\mid p=0.019$ ). No association was found between dynamic airway collapse and symptoms or healthcare utilisation.

Conclusions: This study supports an association between dynamic intra-thoracic central airway collapse and OSA, despite the failure to identify EDAC, which was likely due to retrospectively audited $C T$ scans not being performed to research standards.

Keywords: Obstructive sleep apnoea, Tracheobronchomalacia, Excessive dynamic airway collapse

\footnotetext{
* Correspondence: thomas.crowhurst@gmail.com

The research was presented in abstract form at the Australasian Sleep Association conference in Sydney in October 2019.

'Discipline of Medicine, University of Adelaide, Adelaide, SA 5000, Australia

${ }^{2}$ Central Adelaide Local Health Network, 1 Port Road, Adelaide, SA 5000,

Australia

Full list of author information is available at the end of the article
}

(c) The Author(s). 2020 Open Access This article is licensed under a Creative Commons Attribution 4.0 International License, which permits use, sharing, adaptation, distribution and reproduction in any medium or format, as long as you give appropriate credit to the original author(s) and the source, provide a link to the Creative Commons licence, and indicate if changes were made. The images or other third party material in this article are included in the article's Creative Commons licence, unless indicated otherwise in a credit line to the material. If material is not included in the article's Creative Commons licence and your intended use is not permitted by statutory regulation or exceeds the permitted use, you will need to obtain permission directly from the copyright holder. To view a copy of this licence, visit http://creativecommons.org/licenses/by/4.0/. 


\section{Introduction}

Excessive central airway collapse (ECAC) is caused either by structural deficiency of airway cartilage (tracheobronchomalacia) or by disproportionate bulging of the pars membranosa into the airway lumen (excessive dynamic airway collapse ('EDAC') with preservation of the semicircular configuration of the cartilaginous anterolateral central airway. Although the phenomenon of EDAC has been long recognised, inconsistent terminology and definitions of severity have previously impeded progress. A classification system has been proposed and the threshold for abnormal collapse in healthy volunteers has been more rigorously defined, being $a \geq 75 \%$ reduction in crosssectional airway area (CSA) on spirometry-controlled dynamic CT chest (Murgu \& Colt, 2007; Boiselle et al., 2009; O’Donnell et al., 2012; Litmanovich et al., 2010). Nonetheless understanding of EDAC remains incomplete and, importantly, the association of this condition with other comorbidities and respiratory symptoms remains uncertain (Murg \& Colt, 2013; Matus et al., 2016).

Pathophysiological principles suggest an association should exist between EDAC and obstructive sleep apnoea (OSA). Repetitive tension generated by inspiratory efforts against an occluded upper airway in sleep could promote the development of EDAC. Chronic airway inflammation from associated micro-aspiration could also contribute to reduced elasticity. Only three studies have examined links between the two conditions, with mixed results (Ehtisham et al., 2015; Kolakowski et al., 2018; Good et al., 2018). These studies have important limitations including the conceptualisation and analysis of dynamic intra-thoracic central airway behaviour as a categorical phenomenon, with collapse of the pars membranosa being either 'normal' or 'excessive' but with no gradation in between; this arbitrarily simplifies the inherently continuous nature of dynamic intra-thoracic central airway collapse, overvalues the strength of the research that has defined the threshold for pathological collapse, and limits nuance in what remains an exploratory area of investigation. Potential associations between OSA and degree of dynamic intra-thoracic central airway collapse clearly warrant further study.

We hypothesised that EDAC (as a categorical variable) would be associated with OSA. Additionally, noting the limitations of earlier studies, we hypothesised that degree of dynamic intra-thoracic central airway collapse (as a continuous variable) would be associated with OSA. Separately, we hypothesised that both EDAC and degree of dynamic intra-thoracic central airway collapse would be associated with increased symptom burden and / or healthcare utilisation.

\section{Materials and methods}

\section{Study design}

This multi-centre retrospective audit was approved by the local research ethics committee (Central Adelaide Local Health Network Research Ethics Committee approval number 137). Inclusion criteria were (a) dynamic CT chest for any indication between 1 January 2009 and 31 December 2018 at any public hospital in the state, (b) diagnostic polysomnography (level 1 or 2) within 2 years of the dynamic CT chest and (c) available records for audit.

We excluded patients with known tracheobronchomalacia or risk factors for this separate form of ECAC (being current or previous tracheostomy, compressive lesion, relapsing polychronditis and cystic fibrosis).

\section{Diagnostic investigations Dynamic CT chest}

The retrospective nature of this audit, its chronological duration and the inclusion of multiple sites renders it impossible to describe the technical specifics of each scanner. Dynamic CT chest involved acquisition of images at full inspiration and full expiration. Patients were coached to perform breathing manoeuvres by radiographers, however spirometry-control and respiratory physiologist supervision were not employed.

CTs were reviewed in Picture Archiving Communications System / Radiology Information System (Carestream Health Systems, Version 11.4.1102, Rochester, New York). Fine slice axial inspiratory images were reviewed in lung windows (window: 1500 / level: -600) after multiplanar reconstruction. Following doubleoblique alignment to the axis of the airway, CSA measurements were acquired using the Freehand ROI tool at five locations, being: mid-trachea $(10 \mathrm{~mm}$ above level of aortic arch), lower trachea (10 $\mathrm{mm}$ above level of carina), left main bronchus (10 mm distal to origin), right main bronchus (10 mm distal to origin) and bronchus intermedius (10 mm distal to origin). These measurements were then repeated on expiratory images (fine slice where available) following multiplanar reconstruction and double-oblique alignment as described. The morphology of the posterior tracheal wall at the level of the mid-tracheal measurement was classified as concave, straight or convex to provide a surrogate measure of degree of expiratory effort. All analysis was performed by a radiology registrar after proctoring from a consultant radiologist.

EDAC was defined as a categorical variable (either present or not) and was indicated by a $\geq 75 \%$ dynamic reduction in airway CSA at any of the five central airway sites. Degree of dynamic intra-thoracic central airway collapse was defined as a continuous variable from a minimum of $0 \%$ collapse to a maximum of $100 \%$ 
collapse and was comprised of the mean percentage reduction in airway CSA across all five sites.

\section{Diagnostic polysomnography}

Level 1 diagnostic polysomnograms (PSGs) occurred in accredited overnight attended laboratories with the following: three electroencephalogram (EEG) signals, bilateral electro-oculogram, chin electromyogram (EMG), electrocardiogram, bilateral lower limb EMG, nasal pressure, nasal thermistor, chest and abdominal piezometers, external tracheal microphone, peripheral pulse oximetry and infra-red video recording. Level 2 diagnostic PSGs occurred in unattended home environments and utilised all of the same signals except with the following differences: one EEG signal (with one back-up signal), no thermistor, vibration via nasal cannulae for snore instead of external microphone, automated body position sensing and no video recording. All studies were scored by accredited sleep scientists in accordance with contemporaneous American Academy of Sleep Medicine guidelines and were reported by consultant sleep physicians.

\section{Chart review}

Patient notes were reviewed to collect demographics (age, gender, body mass index [BMI], smoking status), symptoms (dyspnoea, cough, excess mucus production), comorbid conditions (asthma, chronic obstructive pulmonary disease [COPD], gastro-oesophageal reflux disease [GORD]), healthcare utilisation (public emergency department presentations, public hospital inpatient admission days, bronchoscopy, death) and pulmonary function test results. Data chronologically closest to the diagnostic PSG were preferenced.

\section{Statistical analysis}

Binary logistic models were employed to examine associations between the categorical variable of EDAC and OSA. Linear regression models were used to test associations between the continuous variable of degree of dynamic intra-thoracic central airway collapse and OSA.

Indices used to reflect OSA were apnoea hypopnoea index (AHI), supine AHI, respiratory disturbance index (RDI), oxygen $3 \%$ desaturation index (ODI) and average peripheral oxygen saturation $(\mathrm{SpO} 2)$ in sleep.

Unadjusted and adjusted linear regression models were performed. Potential confounders were pre-defined to include age, gender, BMI, asthma, COPD, GORD, postbronchodilator forced expiratory volume in $1 \mathrm{sec}$ (FEV1) as percentage of predicted and post-bronchodilator forced vital capacity (FVC) as percentage of predicted.

Bivariate linear regressions were first performed with one possible confounder in each model. Multivariate linear regression models were then created by including all potential confounders with $P$ value $<0.2$ on bivariate regression. Backwards elimination was then performed, removing the confounder with the highest $P$ value one at a time until all $P$ values were less than 0.05 , thereby giving the most parsimonious and efficient multivariate models.

Binary logistic and negative binomial regressions were performed to assess associations between symptoms and healthcare utilisation and the categorical variable of EDAC and, separately, between the same outcomes and the continuous variable of degree of dynamic intrathoracic central airway collapse. Unadjusted and adjusted regression models were then performed with the adjusted models including those outcomes found to be associated with EDAC in the analysis for OSA as described above.

Indices reflecting symptoms were dyspnoea (World Health Organisation functional class), cough (yes / no) and excess mucus production (yes / no). Indices used to reflect healthcare utilisation were public emergency department presentations (rate per year), public hospital inpatient admission days (days per year), bronchoscopy (yes / no) and death (yes / no).

Linear regression with logarithmic transformation was used to test whether there was a systematic bias in AHI between level 1 and level 2 studies.

$P$ value $<0.05$ was defined apriori as indicating statistical significance. Data were compiled in Excel (Microsoft, version 1905, Redmond, Washington). Statistical analysis was performed using SAS (SAS Institute Incorporated, version 9.4, Cary, North Carolina).

\section{Results}

A total of 61 patients met inclusion criteria but two were excluded (both had cystic fibrosis), leaving 59 patients for assessment. Median age was 62 years (interquartile range [IQR]: $51-70$ years) and 59\% were female. Median BMI was $33 \mathrm{~kg} / \mathrm{m}^{2}$ (IQR: $30-38 \mathrm{~kg} / \mathrm{m}^{2}$ ). Most patients were reformed smokers (27 patients | 46\%) or never smokers (22 patients | 37\%) with a minority actively smoking (10 patients | 17\%). Median pack-year history in active and reformed smokers was 23 pack-years (IQR 16-33 pack-years).

The comorbidities, symptoms, healthcare utilisation and pulmonary function test results are depicted in Table 1 (categorical variables) and Table 2 (ordinal and continuous variables).

All dynamic CT chest scans were performed as part of a high-resolution CT chest protocol with a focus on lung parenchymal disorders as opposed to central airway collapsibility. Overall 20 scans (34\%) were judged to be good quality (morphology of mid-trachea consistent with adequate expiratory effort and an optimal number of slices available on expiratory phase). 
Table 1 Rates of categorical symptoms, comorbidities and healthcare utilisation

\begin{tabular}{lll}
\hline Variable & Number affected & Percentage of total \\
\hline Cough & 12 & $20 \%$ \\
Excess mucus production & 5 & $9 \%$ \\
Asthma & 16 & $27 \%$ \\
COPD & 17 & $29 \%$ \\
GORD & 21 & $36 \%$ \\
Bronchoscopy & 5 & $8 \%$ \\
Death & 14 & $24 \%$ \\
\hline
\end{tabular}

Legend: COPD chronic obstructive pulmonary disease, GORD gastrooesophageal reflux disease

EDAC was not found in any patient. The median degree of dynamic intra-thoracic central airway collapse (quantified by mean percentage reduction in airway CSA on expiration across all five sites in each patient) was 18\% (IQR: $9-30 \%$ | range 0-63\%).

The majority of PSGs (88\%) were level 2 studies. Although level 1 studies provide higher quality data for the assessment of sleep-disordered breathing compared with level 2 studies, linear regression with logarithmic transformation did not demonstrate any statistically significant association between AHI and level of PSG $(P=$ 0.51). Median AHI was 13 events/hour (IQR: 5-31 events/hour | range 0-153 events/hour) and a total of 16 patients $(27 \%)$ had severe OSA when defined as an AHI of $\geq 30$ events/hour. Median supine AHI was 24 events/hour (IQR: 8-70 events/hour). Median RDI was 14 events/hour (IQR: 6-33 events/hour). Median ODI was 16 events/hour (IQR: 6-29 events/hour). Median average $\mathrm{SpO} 2$ in supine sleep was 92\% (IQR: $88-94 \%$ ).

Unadjusted and adjusted linear regression analyses demonstrated statistically significant associations between degree of dynamic intra-thoracic central airway collapse and multiple OSA indices including overall

Table 2 Rates of ordinal and continuous symptoms, healthcare utilisation and pulmonary function test results

\begin{tabular}{lll}
\hline Variable & Median & Interquartile range \\
\hline Dyspnoea & 2 & $2-3$ \\
ED presentations per year & 0 & $0-0.6$ \\
Inpatient admission days per year & 0 & $0-3.8$ \\
FEV1 as percentage of predicted & $70 \%$ & $52-93 \%$ \\
FVC as percentage of predicted & $78 \%$ & $62-98 \%$ \\
TLC as percentage of predicted & $93 \%$ & $75-107 \%$ \\
DLCO as percentage of predicted & $68 \%$ & $55-83 \%$ \\
\hline
\end{tabular}

Legend: Dyspnoea was an ordinal variable with a scale of increasing severity from 1 to 4 (World Health Organisation functional class). ED emergency department, FEV1 forced expiratory volume in 1 second, FVC forced vital capacity, $T L C$ total lung capacity, DLCO diffusing capacity of carbon monoxide corrected for haemoglobin
AHI, RDI, ODI and average SpO2 in supine and nonsupine sleep (Tables 3 and 4). BMI and gender were consistently identified as relevant confounders for inclusion in the adjusted multivariate linear regression models; increased BMI and male gender were associated with increased dynamic intra-thoracic central airway collapse. The Pearson correlation coefficient for the unadjusted linear model associating composite mean percentage dynamic intra-thoracic central airway collapse and overall AHI was $0.419(p=0.0010)$, representing a statistically significant moderately strong association (Fig. 1). In the adjusted model, every 10\% worsening in dynamic airway collapse was associated with an increase in AHI of 5 events/hour (95\% confidence interval: $0.9-10$ events/hour $\mid P=0.019$ ).

No association was demonstrated between degree of dynamic intra-thoracic central airway collapse and symptoms or healthcare utilisation.

\section{Discussion}

Some expiratory narrowing of central airways is physiological. The equal pressure point (EPP) theory is key. Alveolar pressure, the driving pressure for gas movement on expiration, is comprised of elastic recoil pressure plus pleural pressure. Alveolar pressure ('intraluminal' pressure outside alveoli) decreases on movement up the tracheobronchial tree because pressure is required to force gas across airway resistance; this is supported by the Bernoulli principle, which operates due to the decreasing total CSA of airways from periphery to mouth, and which states the perpendicular pressure exerted by a gas decreases as its velocity through a tube increases. Intraluminal pressure also decreases as expiration progresses because elastic recoil falls, and airway resistance increases, as lung volume reduces.

There is a dynamic point along the tracheobronchial tree where the intraluminal pressure falls to become equal to the external pleural pressure (the EPP). The airway is prone to collapse downstream (closer to mouth) of the EPP because pleural pressure can exceed intraluminal pressure. The system functions as a Starling resistor so airway collapse downstream of the EPP does not contribute to airway resistance (West \& Luks, 2016). The EPP usually exists in lobar to subsegmental bronchi and from here to the mouth there is minimal reduction in intraluminal pressure and minimal resistance. EDAC does not reduce expiratory airflow (Kolakowski et al., 2018; Macklem et al., 1965; Macklem \& Wilson, 1965; Smaldone \& Smith, 1985; Loring et al., 2007; Boiselle et al., 2012; Sverzellati et al., 2009).

EDAC may arise from diseases that drive the EPP closer to the alveolus plus, separately, increased collapsibility of the pars membranosa. Diseases with increased airway resistance (asthma and chronic bronchitis) will 
Table 3 Unadjusted linear regression models assessing associations between intra-thoracic central airway collapse and obstructive sleep apnoea

\begin{tabular}{|c|c|c|c|}
\hline OSA index & $\begin{array}{l}\text { Expected change in OSA index with a 10\% increase in dynamic intra-thoracic central } \\
\text { airway collapse }\end{array}$ & $\begin{array}{l}95 \% \text { confidence } \\
\text { interval }\end{array}$ & $P$ value \\
\hline$\overline{\mathrm{AHI}}$ & 9.1 events/hour & $\begin{array}{l}4.1 \text { to } 14.1 \text { events/ } \\
\text { hour }\end{array}$ & 0.0004 \\
\hline Supine $A H I$ & 9.5 events/hour & $\begin{array}{l}1.4 \text { to } 17.5 \text { events/ } \\
\text { hour }\end{array}$ & 0.0211 \\
\hline $\mathrm{AHI}$ in REM sleep & 0.7 events/hour & $\begin{array}{l}-4.3 \text { to } 5.7 \text { events/ } \\
\text { hour }\end{array}$ & 0.7736 \\
\hline $\mathrm{RDI}$ & 9.2 events/hour & $\begin{array}{l}4.0 \text { to } 14.3 \text { events/ } \\
\text { hour }\end{array}$ & 0.0006 \\
\hline ODI & 15.0 events/hour & $\begin{array}{l}8.5 \text { to } 21.6 \text { events/ } \\
\text { hour }\end{array}$ & $<0001$ \\
\hline $\begin{array}{l}\text { Average } \mathrm{SpO} 2 \text { in supine } \\
\text { sleep }\end{array}$ & $-1.8 \%$ & -0.8 to $-2.8 \%$ & 0.0006 \\
\hline $\begin{array}{l}\text { Average } \mathrm{SpO} 2 \text { in non-supine } \\
\text { sleep }\end{array}$ & $-1.3 \%$ & -0.4 to $-2.2 \%$ & 0.0055 \\
\hline
\end{tabular}

Legend: Associations are described by the expected change in the obstructive sleep apnoea index given a $10 \%$ increase in dynamic intra-thoracic central airway collapse. OSA obstructive sleep apnoea, $A H I$ apnoea hypopnoea index, REM rapid eye movement sleep, $R D I$ respiratory disturbance index, ODI oxygen $3 \%$ desaturation index, $\mathrm{SpO} 2$ peripheral oxygen saturation

precipitate a more rapid fall in intraluminal pressure and thus drive the EPP further upstream. Loss of elastic recoil (emphysema) does the same by reducing driving pressure for gas movement and, separately, diminishing tractional forces that promote airway patency. Obesity increases pleural pressure and forces the individual to respire at reduced lung volumes where elastic recoil is lower and airway resistance is higher (Behazin et al., 1985). Conversely there are limited data to identify which diseases promote increased intrinsic collapsibility of the pars membranosa. These could include obesity (increased extra-luminal pressure and mediastinal fat), chronic cough (repetitive tension, micro-trauma and reduced elasticity), OSA (repetitive tension from inspiratory efforts against occluded upper airway) and chronic airway inflammation (reduced elasticity) from any cause be it intrinsic or extrinsic (Kolakowski et al., 2018; Good et al., 2018). Indeed Majid et al. found $45 \%$ of 139 patients with ECAC had GORD (Majid et al., 2019).

Defining a 'pathological' threshold for distinguishing normal dynamic central airway narrowing from EDAC is problematic. Historically a $50 \%$ reduction in airway CSA was set. Subsequent studies with spirometry-controlled dynamic CT scans with physiologist coaching have

Table 4 Adjusted linear regression models assessing associations between intra-thoracic central airway collapse and obstructive sleep apnoea

\begin{tabular}{|c|c|c|c|c|}
\hline OSA index & $\begin{array}{l}\text { Confounders in } \\
\text { adjusted model }\end{array}$ & $\begin{array}{l}\text { Expected change in OSA index with a } 10 \% \text { increase in dynamic } \\
\text { intra-thoracic airway collapse }\end{array}$ & $\begin{array}{l}95 \% \text { confidence } \\
\text { interval }\end{array}$ & $\begin{array}{l}\mathrm{P} \\
\text { value }\end{array}$ \\
\hline$\overline{\mathrm{AHI}}$ & Gender, BMl & 5.4 events/hour & $\begin{array}{l}0.9 \text { to } 10 \text { events/ } \\
\text { hour }\end{array}$ & 0.0187 \\
\hline Supine AHI & Gender, BMI & 3.8 events/hour & $\begin{array}{l}-3.5 \text { to } 11.1 \\
\text { events/hour }\end{array}$ & 0.3129 \\
\hline AHI in REM sleep & $\mathrm{BMl}$ & -0.5 events/hour & $\begin{array}{l}-5.4 \text { to } 4.4 \\
\text { events/hour }\end{array}$ & 0.84 \\
\hline $\mathrm{RDI}$ & Gender, BMl & 5.5 events/hour & $\begin{array}{l}0.8 \text { to } 10.2 \\
\text { events/hour }\end{array}$ & 0.0230 \\
\hline ODI & Gender, BMl & 10.6 events/hour & $\begin{array}{l}4.5 \text { to } 16.6 \\
\text { events/hour }\end{array}$ & 0.0006 \\
\hline $\begin{array}{l}\text { Average } \mathrm{SpO} 2 \text { in supine } \\
\text { sleep }\end{array}$ & $\mathrm{BMl}$ & $-1.5 \%$ & -0.6 to $-2.5 \%$ & 0.001 \\
\hline $\begin{array}{l}\text { Average } \mathrm{SpO} 2 \text { in non- } \\
\text { supine sleep }\end{array}$ & FEV1 & $-1.2 \%$ & -0.4 to $-2.0 \%$ & 0.0038 \\
\hline
\end{tabular}

Legend: Associations are described by the expected change in the obstructive sleep apnoea index given a $10 \%$ increase in dynamic intra-thoracic central airway collapse. OSA obstructive sleep apnoea, $A H I$ apnoea hypopnoea index, $B M I$ body mass index, $R E M$ rapid eye movement sleep, $R D I$ respiratory disturbance index, $O D I$ oxygen $3 \%$ desaturation index, SpO2 peripheral oxygen saturation, FEV1 forced expiratory volume in $1 \mathrm{sec}$ 


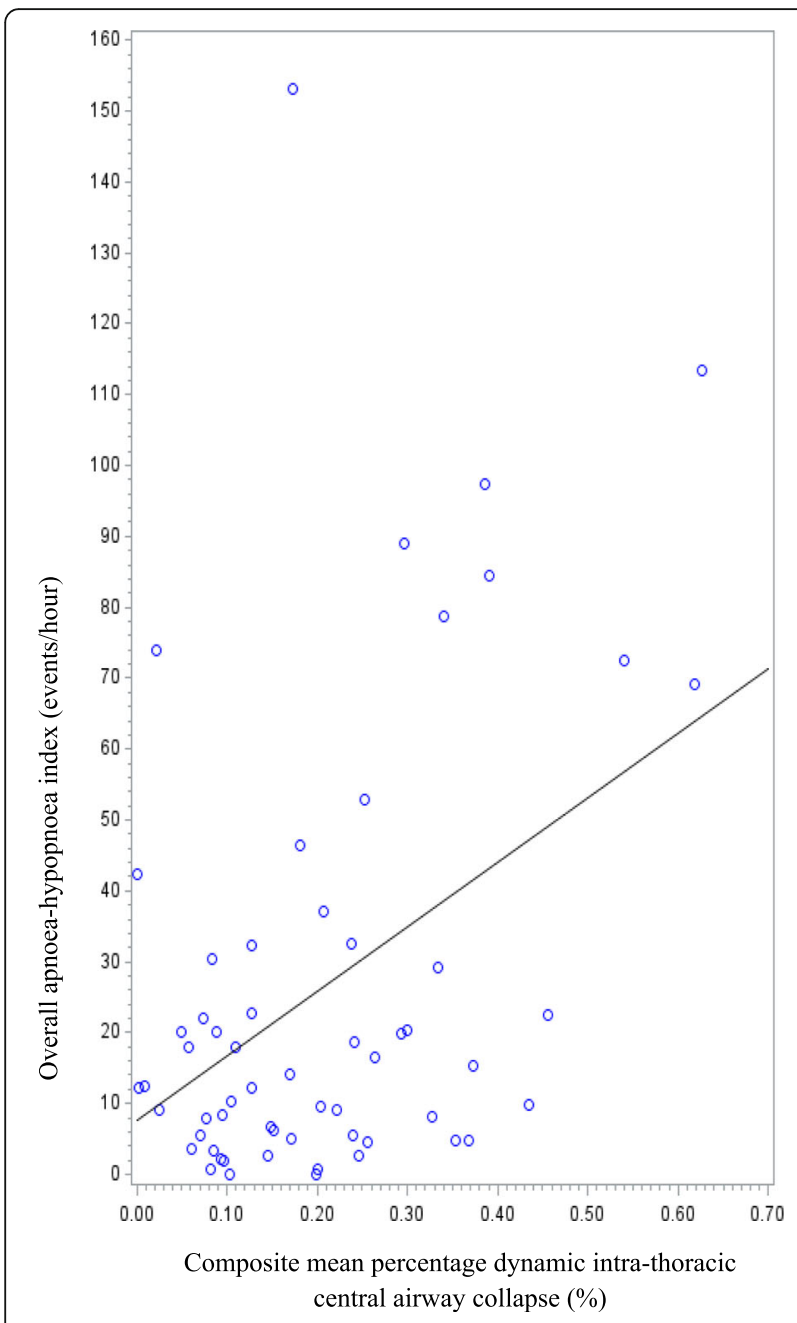

Fig. 1 Scatterplot demonstrating the association between dynamic intra-thoracic central airway collapse and overall apnoea-hypopnoea index. Legend: Composite mean percentage dynamic intra-thoracic central airway collapse is on the $\mathrm{X}$ axis and overall apnoea-

hypopnoea index is on the $Y$ axis, with the unadjusted linear model depicted by the solid black line (Pearson correlation coefficient 0.419 and with $p=0.0010$ )

demonstrated healthy controls have approximately 50 $60 \%$ reduction in CSA with a $20 \%$ standard deviation (Boiselle et al., 2009; O'Donnell et al., 2012; Litmanovich et al., 2010; Boiselle et al., 2012). Thus 75\% collapse may be a superior threshold for defining EDAC, provided imaging is performed with the same robust methods. However such scans are not readily available in most institutions and, furthermore, it is critical to appreciate that this definition of EDAC arises from limited assessments of healthy volunteers and has not been rigorously examined in symptomatic patients or in those with underlying lung disease (Murg \& Colt, 2013; Kolakowski et al., 2018). Differential thresholds of 'pathological' central airway collapse may be applicable in different patient groups. Investigation of dynamic intra-thoracic central airway collapse as a continuous rather than a categorical variable may help to address these issues.

Other methods of assessment of dynamic intrathoracic central airway behaviour have limitations that likely outweigh those associated with dynamic CT imaging. Bronchoscopy allows direct real-time visualisation of the intra-thoracic central airways however precise measurement of airway collapse is hampered by (a) parallax error and other technical factors that can impede CSA calculation, (b) effects of sedation which may alter airway behaviour when compared with wakefulness, (c) continuous positive pressure transmitted to the central airways if nasal high-flow oxygen is utilised, thereby falsely reducing any dynamic collapse, (d) inability to inspect all airway locations simultaneously and (e) increased cost and risk. Although other methods such as drug-induced sleep endoscopy and magnetic resonance imaging have value in examining upper airway function in OSA, they have not been rigorously assessed in dynamic intra-thoracic central airway collapse (Ciscar et al., 2001; Moon et al., 2010; Cavaliere et al., 2013; Kim et al., 2014; Certal et al., 2016; Feng et al., 2018).

A disease should have attributable symptoms or sequalae and here, as outlined previously, EDAC remains particularly poorly characterised. Boiselle and colleagues examined degree of dynamic central airway collapse in 100 patients with COPD and found only weak correlation with symptoms (Boiselle et al., 2012). Good et al. found $76 \%$ of 42 patients with chronic cough had $\geq 65 \%$ dynamic central airway collapse, providing indirect support for the common belief that central airway collapse contributes to cough while also revealing the potential pitfalls of fixating on $\geq 75 \%$ reduction in CSA as the only clinically relevant threshold for pathological dynamic central airway collapse (Good et al., 2018). No studies have thoroughly investigated associations between degree of dynamic intra-thoracic central airway collapse and respiratory complications or mortality.

Existing evidence for an association between EDAC and OSA is mixed. Ehtisham et al. reported a retrospective case series in which $62 \%$ of 107 patients with ECAC had OSA, but neither degree of airway collapse required for EDAC diagnosis nor OSA severity were specified (Ehtisham et al., 2015). Good et al. found OSA (defined as AHI of $\geq 5$ events/hour) in $78 \%$ of 32 patients with both chronic cough and $\geq 65 \%$ dynamic change in central airway CSA, being their threshold for significant collapse (Good et al., 2018). Kolakowski and colleagues audited 200 patients who had undergone investigations for both EDAC (defined as $\geq 75 \%$ collapse) and OSA (Kolakowski et al., 2018). Patients were subdivided into those with and without OSA (defined as AHI of $\geq 5$ events/hour). Prevalence of EDAC across both groups was approximately $25 \%$ but severe EDAC (arbitrarily 
defined in their study as $\geq 85 \%$ collapse) occurred significantly more often in patients with OSA (15\% versus 6\%). Degree of dynamic tracheal collapse was also assessed as a continuous variable, being maximal percentage CSA reduction at any location in the trachea, and this was weakly correlated with supine but not overall AHI. Although the work by Kolakowski et al. is valuable in this developing area, their analysis also exemplifies the limitations that can arise from a categorical statistical methodology; in their research this was applied not only to the degree of dynamic intra-thoracic central airway collapse but also OSA, which is another complex and inherently continuous pathophysiological phenomenon.

Our study adds significantly to the existing literature because it is the first to demonstrate statistically robust associations between degree of dynamic intra-thoracic central airway collapse and multiple OSA indices reflecting both respiratory event-related sleep fragmentation and hypoxic burden. An important strength of our study is the introduction of a multi-site continuous composite index of airway collapse rather than the sole use of the arbitrary categorical presence of 'EDAC' at any one site, thereby more accurately reflecting complex central airway behaviour; this novel approach permitted our analysis to elucidate, for the first time, the fractional impact of increasing dynamic intra-thoracic central airway collapse on upper airway collapsibility in sleep. This is also the first study to utilise regression models to adjust for confounders, which is important when diseases may share risk factors.

We stress that we do not claim to have proven an association between EDAC, as defined as a $\geq 75 \%$ reduction in airway CSA, and OSA; rather, our data support the theory that increasing dynamic intra-thoracic central airway collapse is associated with increasingly severe obstructive sleep apnoea, as measured by obstructive respiratory events and hypoxic burden. Failure to identify EDAC in our study was likely because the dynamic CT chest scans were all performed as part of high-resolution protocols with a focus on lung parenchyma rather than airway collapse, meaning there was none of the spirometry-control or physiologist coaching used in the research protocols that identified $\geq 75 \%$ collapse as abnormal in healthy volunteers. This methodological flaw was unavoidable in a retrospective study and explains why only $34 \%$ of scans were graded as good quality for our purposes (evidence of adequate expiratory effort and optimal number of slices on expiratory phase). However, if anything, any bias arising from this weakness would have reduced the probability of finding a relationship between dynamic intra-thoracic central airway collapse and OSA. Future prospective research with dedicated high-quality dynamic CT imaging is required to further explore the potential association.
Analysis of any association between airway collapse and symptoms / healthcare utilisation would have been confounded by the inclusion of dynamic CT chest scans for any indication, meaning all patients must have had some respiratory symptom to prompt investigation.

\section{Conclusions}

This study provides compelling evidence for a relationship between dynamic intra-thoracic central airway collapse and OSA especially because, even after adjusting for confounders, multiple OSA indices continued to demonstrate statistically and clinically significant associations with degree of collapse. We feel that these findings should prompt clinicians to consider EDAC in patients with severe OSA and vice versa. The shared phenotype may be the obese middle-aged patient with underlying airways disease and chronic cough, although further work is clearly required to test these commonly held clinical beliefs. Continuous positive airway pressure offers the potential to improve both conditions while mucus retention due to EDAC may benefit from positive expiratory pressure techniques for sputum clearance (Murgu \& Colt, 2007; Dedhia et al., 2015; Adliff et al., 1997; Sirithangkul et al., 2010).

Future research could seek to prospectively confirm the association and test its extension to EDAC, clarify any association between airway collapse and symptoms / healthcare utilisation, assess whether differing thresholds of 'pathological' airway collapse may be applicable in different patient populations, and determine if physiological tests like impulse oscillometry can identify EDAC with or without OSA (Fielding et al., 2018).

\section{Abbreviations \\ AHI: Apnoea-hypopnoea index; COPD: Chronic obstructive pulmonary disease; CPAP: Continuous positive airway pressure; CSA: Cross-sectional area; $\mathrm{CT}$ : Computed tomography; ECAC: Excessive central airway collapse; EDAC: Excessive dynamic airway collapse; EPP: Equal pressure point; FEV1: Forced expiratory volume in 1 second; FVC: Forced vital capacity; GORD: Gastro-oesophageal reflux disease; IQR: Inter-quartile range; ODI: Oxygen 3\% desaturation index; OSA: Obstructive sleep apnoea; RDI: Respiratory disturbance index; REM: Rapid eye movement; SpO2: Peripheral oxygen saturation}

\section{Acknowledgements}

The authors sincerely thank Suzanne Edwards for her generous assistance with statistical design and analysis. The authors thank Dr. Mark Jurisevic and Dr. Sonya Johnston for assistance in collating polysomnography data.

\section{Authors' contributions}

Dr. Crowhurst conceptualised the study, developed the methodology, coordinated data collection and drafted the manuscript; he is guarantor for the paper, taking responsibility for the integrity of the work as a whole from inception to submission for publication. Dr. Tcherveniakov contributed significantly to the methodology, particularly relating to imaging analysis, collected and analysed data and reviewed the manuscript. Dr. Lorraine contributed significantly to the methodology, particularly relating to imaging analysis, and reviewed the manuscript. Dr. Polasek contributed to the methodology, assisted in data access and reviewed the manuscript. A/Prof Nguyen assisted in conceptualisation of the study, contributed to the methodology and reviewed the manuscript. Dr. Yeo assisted in conceptualisation of the study, 
contributed to the methodology, assisted in data analysis and reviewed the manuscript. The author(s) read and approved the final manuscript.

\section{Funding}

No external funding was received for this study.

\section{Availability of data and materials}

All data generated or analysed during this study are included in this published article.

\section{Ethics approval and consent to participate}

This multi-centre retrospective audit was approved by the local research ethics committee (Central Adelaide Local Health Network Research Ethics Committee approval number 137). The requirement for patient consent was waived by the ethics committee given the retrospective nature of the research involving existing clinical information and the negligible risk to patients.

\section{Consent for publication}

Not applicable.

\section{Competing interests}

The authors declare that they have no completing interests.

\section{Author details}

'Discipline of Medicine, University of Adelaide, Adelaide, SA 5000, Australia. ${ }^{2}$ Central Adelaide Local Health Network, 1 Port Road, Adelaide, SA 5000, Australia. ${ }^{3}$ Department of Thoracic Medicine, Royal Adelaide Hospital, 1 Port Road, Adelaide, SA 5000, Australia.

Received: 26 December 2019 Accepted: 30 April 2020

Published online: 16 June 2020

\section{References}

Adliff M, Ngato D, Keshavjee $S$, et al. Treatment of diffuse tracheomalacia secondary to relapsing polychronditis with continuous positive airway pressure. Chest. 1997:112(6):1701-4.

Behazin N, Jones S, Cohen R, et al. Respiratory restriction and elevated pleural and esophageal pressures in morbid obesity. J Appl Physiol. 1985;108(1):212-

Boiselle P, Michaud G, Roberts D, et al. Dynamic expiratory tracheal collapse in COPD: correlation with clinical and physiologic parameters. Chest. 2012; 142(6):1539-44

Boiselle P, O'Donnell C, Bankier A, et al. Tracheal collapsibility in healthy volunteers during forced expiration: assessment with multi-detector $\mathrm{CT}$. Radiology. 2009;252(1):255-62.

Cavaliere M, Russo F, lemma M, et al. Awake versus drug-induced sleep endoscopy: evaluation of airway obstruction in obstructive sleep apnea / hypopnoea syndrome. Laryngoscope. 2013;123(9):2315-8.

Certal V, Pratas R, Guimaraes $L$, et al. Awake examination versus DISE for surgical decision making in patients with OSA: a systematic review. Laryngoscope. 2016;126(3):768-74.

Ciscar M, Juan G, Martinez V, et al. Magnetic resonance imaging of the pharynx in OSA patients and healthy subjects. Eur Respir J. 2001;17(1):79-86.

Dedhia R, Kapur V, Weaver E. Excessive dynamic airway collapse of the lower airway: a cause for persistent sleep-disordered breathing after tracheostomy. J Clin Sleep Med. 2015:11(11):1337-9.

Ehtisham M, Azhar-Munir R, Klopper E, et al. Correlation between tracheobronchomalacia / hyper dynamic airway collapse and obstructive sleep apnoea. Am J Respir Crit Care Med. 2015;191:A3075.

Feng $Y$, Keenan B, Wang S, et al. Dynamic upper airway imaging during wakefulness in obese subjects with and without sleep apnea. Am J Respir Crit Care Med. 2018:198(11):1435-43.

Fielding D, Travers J, Nguyen P, et al. Expiratory reactance abnormalities in patients with expiratory dynamic airway collapse: a new application of impulse oscillometry. ERJ Open Res. 2018:4(4):00080-2018. https://doi.org/10. 1183/23120541.00080-2018

Good J, Rollins D, Kolakowski C, et al. New insights in the diagnosis of chronic refractory cough. Respir Med. 2018;141:103-10.

Kim A, Keenan B, Jackson N, et al. Tonque fat and its relationship to obstructive sleep apnea. Sleep. 2014;37(10):1639-48.
Kolakowski C, Rollins D, Jennermann T, et al. Clarifying the link between sleep disordered breathing and tracheal collapse: a retrospective analysis. Sleep Sci Pract. 2018;2:10. https://doi.org/10.1186/s41606-018-0030-2.

Litmanovich D, O'Donnell C, Bankier A. Bronchial collapsibility at forced expiration in healthy volunteers: assessment with multidetector $C T$. Radiology. 2010;257(2):560-7.

Loring S, O'Donnell C, Feller-Kopman D, et al. Central airway mechanics and flow limitation in acquired tracheobronchomalacia. Chest. 2007;131(4):1118-24.

Macklem P, Fraser R, Brown W. The detection of the flow-limiting bronchi in bronchitis and emphysema by airway pressure measurements. Med Thorac. 1965:22:220-30.

Macklem P, Wilson N. Measurement of intrabronchial pressure in man. J Appl Physiol. 1965;20(4):653-63.

Majid A, Kheir F, Alape D, et al. The prevalence of gastroeosophageal reflux in patients with excessive central airway collapse. Chest. 2019;155(3):540-5.

Matus I, Richter W, Mani S. Awareness, competencies, and practice patterns in tracheobronchomalacia: a survey of pulmonologists. J Bronchology Interv Pulmonol. 2016;23(2):131-7.

Moon I, Han D, Kim J, et al. Sleep magnetic resonance imaging as a new diagnostic method in obstructive sleep apnea syndrome. Laryngoscope. 2010;120(12):2546-54.

Murg S, Colt H. Tracheobronchomalacia and excessive dynamic airway collapse. Clin Chest Med. 2013;34(3):527-55.

Murgu S, Colt H. Description of multidimensional classification system for patients with expiratory central airway collapse. Respirology. 2007;12(4):54350.

O'Donnell C, Litmanovich D, Loring S, et al. Age and sex dependence of forced expiratory central airway collapse in healthy volunteers. Chest. 2012;142(1): $168-74$.

Sirithangkul S, Ranganathan S, Robinson P, et al. Positive expiratory pressure to enhance cough effectiveness in tracheomalacia. J Med Assoc Thail. 2010; 93(6):S122-8.

Smaldone G, Smith P. Location of flow-limiting segments via airway catheters near residual volume in humans. J Appl Physiol. 1985;59(2):502-8.

Sverzellati N, Rastelli A, Chetta A. Airway malacia in chronic obstructive pulmonary disease: prevalence, morphology and relationship with emphysema, bronchiectasis and bronchial wall thickening. Eur Radiol. 2009, 19(7):1669-78.

West J, Luks A. West's respiratory physiology: the essentials. Philadelphia, PA: Wolters Kluwer; 2016

\section{Publisher's Note}

Springer Nature remains neutral with regard to jurisdictional claims in published maps and institutional affiliations.

Ready to submit your research? Choose BMC and benefit from:

- fast, convenient online submission

- thorough peer review by experienced researchers in your field

- rapid publication on acceptance

- support for research data, including large and complex data types

- gold Open Access which fosters wider collaboration and increased citations

- maximum visibility for your research: over $100 \mathrm{M}$ website views per year

At $\mathrm{BMC}$, research is always in progress.

Learn more biomedcentral.com/submission 